\title{
ANALISIS CUANTITATIVO Y CUALITATIVO DE LA AEROMICOTA AISLADA DE LA CATEDRAL DE SANTIAGO DE COMPOSTELA(GALICIA, ESPAÑA)
}

\author{
(Quantitative and qualitative analysis of the airborne fungi isolated in the \\ Cathedral of Santiago de Compostela (Galicia, Spain))
}

* M.J. Aira; **Rodríguez-Rajo, F.J. ; **Jato, V. \& *** Piontelli, E.

*Departamento de Botánica, Facultad de Farmacia, Universidad de Santiago (España)

**Departamento de Biología Vegetal y Ciencias del Suelo, Facultad de Ciencias de Ourense, Universidad de Vigo (España) *** Cátedra de Micología, Escuela de Medicina,

Universidad de Valparaíso (Chile)

Palabras clave: hongos, anemófilos, Aspergillus fumigatus, Penicillium purpurogenum Catedral, Galicia, España. Key words: Anemophilous fungi, Aspergillus fumigatus, Penicillium purpurogenum, Cathedral, Galicia, Spain.

\section{RESUMEN}

Mediante un estudio de la presencia de mitosporas fúngicas atmosféricas en el interior y exterior de la Catedral de Santiago de Compostela (España) durante un año, a través de un sistema de captación volumétrico viable, se identificaron 28 taxa, principalmente: Alternaria, Aspergillus, Cladosporium y Penicillium. Desde el punto de vista cuantitativo no existen diferencias significativas entre interior/exterior, $y$ en ambos casos las concentraciones son ligeramente más elevadas en primavera-verano. Las especies más abundantes fueron: Penicillium purpurogenum Stoll, con un $94 \%$ en la atmósfera interna y un $82 \%$ en el exterior y Aspergillus fumigatus Fres., con un 65\% y un $63 \%$ respectivamente.

\section{INTRODUCCION}

La Catedral de Santiago de Compostela (Galicia, España), está considerada como una de las mejores representaciones del románico español, aunque su construcción, dilatada durante varios siglos, abarca estilos diferentes. Desde el siglo IX y hasta nuestros días, la ciudad y este monumento arquitectónico, es visitado anualmente por numerosos peregrinos (más de 5 millones/año en la última década), ya que es el destino final de una de las rutas más importantes del cristianismo conocida como «el camino de Santiago». El complejo arquitectónico forma

\begin{abstract}
A study was carried out on the atmospheric fungal content in the Cathedral of Santiago de Compostela (Spain) and its exterior during a year, using a viable volumetric collecting system. A total of 28 taxa were identified, the most abundant of which were: Alternaria, Aspergillus, Cladosporium and Penicillium. In quantitative terms there were no significant indoor/ outdoor differences, and the concentrations were slightly higher in Spring-Summer in both cases. The most abundant species were Penicillium purpurogenum Stoll, with $94 \%$ in the indoor atmosphere and $82 \%$ outdoors, and Aspergillus fumigatus Fres., representing 65\% in the indoor and 63\% outdoors.
\end{abstract}

parte del Patrimonio Cultural de la Humanidad señalado por la UNESCO, pese a lo cual, apenas se han realizado estudios orientados a su conservación (Stchigel et al., 2002).

Los contaminantes atmosféricos inorgánicos que afectan a los monumentos urbanos son bien conocidos y las alteraciones que producen en la piedra son muy variadas, grietas y fisuras, eflorescencias, pátinas, costras, oxidaciones, manchas de humedad, etc. (Saiz-Jimenez et al., 1999), pero también es importante la acción de los agentes biológicos como los musgos, líquenes, hongos e incluso algunas bacterias (Gorbushina et al., 2004), además del daño que pueden causar algunos animales sobre todo en las paredes externas (ej.los excrementos de las aves). 
El biodeterioro se puede definir como un cambio indeseable en las propiedades de un determinado material, causado por la actividad de diversos organismos. Está ligado a las condiciones microclimáticas sobretodo a la temperatura y la humedad relativa y puede llevar a su destrucción (Vaillant \& Valentín, 1996). Los materiales que pueden ser alterados por los hongos y bacterias son muy variados: como el papel, madera, tejido, pergamino, etc. Ciertos hongos, como Aureobasidium o Acremonium, son capaces de obtener elementos esenciales para su crecimiento, como el fósforo y el nitrógeno, a partir de vidrio alterado (Gorbushina \& Palinska, 1999). Diversos autores han contribuido al estudio de los hongos que deterioran las vidrieras históricas (Koestler \& Santoro, 1987), el material audiovisual (Tapia, 2006), las bibliotecas y archivos (Singh et al., 1995; Medina et al., 1999), o los restos óseos de colecciones de interés (Calderón, 2004).

La Catedral de Santiago está construida en piedra granítica, una roca de origen magmático (compuesta por cuarzo, feldespato y mica) y en su interior alberga numerosas tallas de madera cubiertas de textil, retablos y sillería, además de los objetos religiosos de vidrio y diferentes tipos de metal.

En el presente trabajo se ha efectuado un análisis anual de la aeromicota en el interior de la Catedral, con el fin de valorar su abundancia e identificar los agentes que podrían producir ciertas alteraciones del propio edificio así como de las obras que alberga, como medida previa a una propuesta de intervención de tipo conservativo. Este estudio, permitirá principalmente valorar la calidad del aire del interior del templo y detectar la presencia de hongos de importancia en salud pública.

\section{MATERIALES Y METODOS}

El presente estudio se llevó a cabo en la Catedral de Santiago de Compostela (Galicia, España) durante todo el año 2002. La recogida de muestras se realizó con un Burkard portable air for agar plates (BPC), que permite la incorporación de una placa con medio de cultivo.

Con el fin de conocer la cantidad y diversidad fúngica a lo largo del año, se realizó un muestreo cada cuatro semanas en cinco puntos de la nave de la Catedral, cuatro en los extremos de la planta de cruz latina (señalados de 1 a 4) y uno en la parte central de la misma (punto 5), realizándose además un muestreo en el exterior del recinto (punto 6), para utilizarlo como referencia del ambiente externo (Figura 1).

Los días elegidos para el muestreo fueron los viernes y los domingos, durante 13 semanas en el año. El viernes se tomó como día representativo de una actividad normal en el recinto, es decir, sin afluencia masiva de gente que pudiese afectar al contenido fúngico atmosférico. El

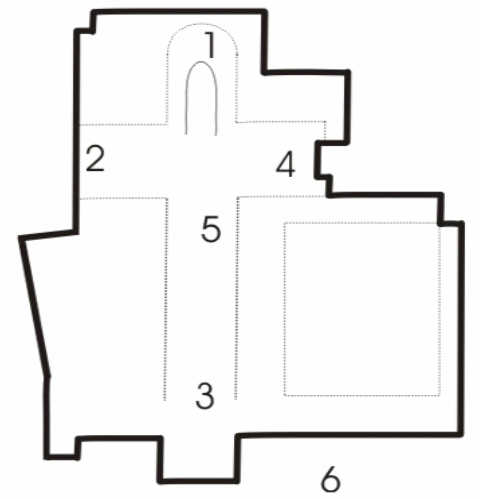

Figura 1. Plano de la Catedral de Santiago (1 Quintana, 2 Azabachería, 3 Obradoiro, 4 Platerías 5 Nave central, 6 Exterior)

muestreo comenzó a las $10 \mathrm{~h}$ de la mañana colocando el BPC en cada uno de los cinco puntos de muestreo (1 a 5) y en el exterior (punto 6), durante 10 minutos, utilizando como medio de cultivo agar Sabouraud cloranfenicol comercial. Además este mismo día, se realizó un muestreo en la zona central del interior de la Catedral (punto 5) en las mismas condiciones que los anteriores, variando únicamente el medio de cultivo, usando Malt Extract Agar (MEA) y el Oat Agar (OA) para cubrir un posible mayor espectro de taxa.

Cada domingo la Catedral puede llegar a reunir a más de 5.000 personas en un servicio religioso. En este caso, el muestreo se llevó a cabo también durante 10 minutos y con Sabouraud cloranfenicol comercial como medio de cultivo, pero a distintas horas del día (9h., 13h. y 21h.), tanto en el interior de la Catedral (punto 5) como en el exterior (punto 6).

En total se han obtenido 182 placas de cultivo, 130 tomadas en el interior y 52 correspondientes al ambiente externo. Todas ellas se han incubado en estufa a $25^{\circ} \mathrm{C}$ durante 7 días, realizando entonces el recuento de las colonias y los correspondientes aislamientos.

Tabla 1.- Características del muestreo realizado los viernes en los puntos 1 al 6 a las 10 horas.

\begin{tabular}{l|c}
\hline Viernes & Medio \\
\hline 25 enero & \\
22 febrero & \\
22 marzo & \\
19 abril & Sabouraud 1,2,3,4,5 y exterior \\
17 Mayo & (6)MEA y OA en punto 5 \\
14 junio & \\
12 julio & \\
9 agosto & \\
6 septiembre & \\
4 octubre & \\
1 y 29 noviembre &
\end{tabular}


Tabla 2.- Características del muestreo realizado los domingos en los puntos 5 y 6 a las 9, 13 y 21 horas.

\begin{tabular}{l|l}
\multicolumn{1}{c|}{ Domingos } & Medio \\
\hline 27 enero & \\
24 febrero & \\
24 marzo & \\
21 abril & \\
19 mayo & \\
16 junio & Sabouraud \\
14 julio & \\
11 agosto & \\
8 septiembre & \\
6 octubre & \\
3 noviembre & \\
1 y 29 diciembre & \\
\hline
\end{tabular}

De forma simultánea, en todos los muestreos realizados, se llevó a cabo la medición de temperatura y humedad por medio de una estación meteorológica weatherlink, anotando también los días de lluvia por su posible influencia en el contenido fúngico de la atmósfera exterior. Por otra parte, se registró la afluencia de personas en la Catedral durante los muestreos, fijando tres categorías: (A) si la cantidad de visitantes era elevada e intermitente, (B) si el número de personas era considerable pero constante y (C) si la presencia era escasa o nula.

\section{RESULTADOS}

El valor promedio de unidades formadoras de colonias contabilizadas en cada uno de los puntos de muestreo de la atmósfera interior de la Catedral (1 a 5) y en el exterior (punto 6), no muestran grandes diferencias, con valores próximos a las $400 \mathrm{CFU} / \mathrm{m}^{3}$ (Figura 2).

El rango de variación osciló entre $70 \mathrm{CFU} / \mathrm{m}^{3}$, contabilizadas el 19 de abril en el punto 2 a las 10 h. y 630 $\mathrm{CFU} / \mathrm{m}^{3}$ en el punto 5 a las $13 \mathrm{~h}$., mientras que en el exterior el valor más bajo fue de $85 \mathrm{CFU} / \mathrm{m}^{3}$ el 19 de abril a las $13 \mathrm{~h}$. y el más elevado de $650 \mathrm{CFU} / \mathrm{m}^{3}$ el 14 de julio a las $9 \mathrm{~h}$.

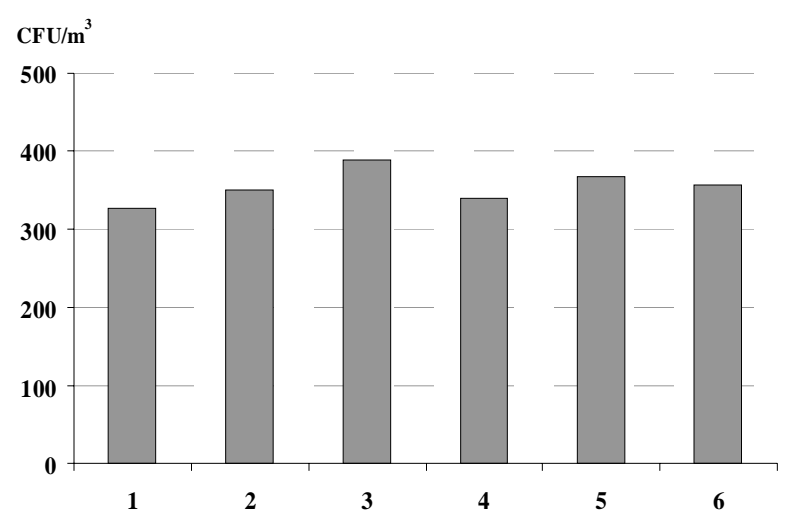

Figura 2.- Promedio de $\mathrm{CFU} / \mathrm{m}^{3}$ en los distintos puntos de muestreo
La concentración de propágulos fúngicos a lo largo del año, es más elevada durante la primavera-verano (Figura 3), disminuyendo considerablemente a partir del mes de septiembre, tanto en el interior del edificio como en el ambiente externo.

En cuanto a la valoración de los medios de cultivo, tampoco se encontraron diferencias muy marcadas, ya que en Malta Extract Agar (MEA) se registró un promedio de $389 \mathrm{CFU} / \mathrm{m}^{3}$ seguido de Sabouraud con un promedio de $366 \mathrm{CFU} / \mathrm{m}^{3}$ y OA con $308 \mathrm{CFU} / \mathrm{m}^{3}$.

Por su parte, el estudio de la representatividad intradiurna realizado en el interior de la Catedral, pone de manifiesto una mayor abundancia de las concentraciones

Tabla 3.- Presencia de taxa identificados por métodos de captación volumétrica

\begin{tabular}{|l|c|c|}
\hline & Catedral & Exterior \\
\hline Acremonium spp. & + & + \\
\hline Alternaria alternata & + & + \\
\hline Aspergillus candidus & + & + \\
\hline Aspergillus flavus & + & + \\
\hline Aspergillus fumigatus & + & + \\
\hline Aspergillus niger & + & \\
\hline Aspergillus oryzae & + & \\
\hline Aspergillus terreus & + & \\
\hline Aspergillus versicolor & + & + \\
\hline Otros Aspergillus & + & + \\
\hline Beauveria bassiana & & + \\
\hline Botrytis cinerea & + & + \\
\hline Chaetomium globosum & + & + \\
\hline Chaetomium indicum & + & + \\
\hline Cladosporium spp. & + & + \\
\hline Cylindrocarpon spp. & + & + \\
\hline Mucor mucedo & + & + \\
\hline Paecilomyces lilacinus & + & + \\
\hline Paecilomyces variotii & + & + \\
\hline Penicillium crustosum & + & + \\
\hline Penicillium funiculosum & + & + \\
\hline Penicillium glabrum & + & + \\
\hline Penicillum pinophyllum & & + \\
\hline Penicillium purpurogenum & + & + \\
\hline Penicillium spinulosum & & + \\
\hline Penicillium thomii & + & + \\
\hline Otros Penicillium & + & + \\
\hline Rhizopus stolonifer & + & + \\
\hline Trichoderma & + & + \\
\hline Trichoderma viride & + & + \\
\hline
\end{tabular}




\section{$\mathbf{C F U} / \mathbf{m}^{3}$}

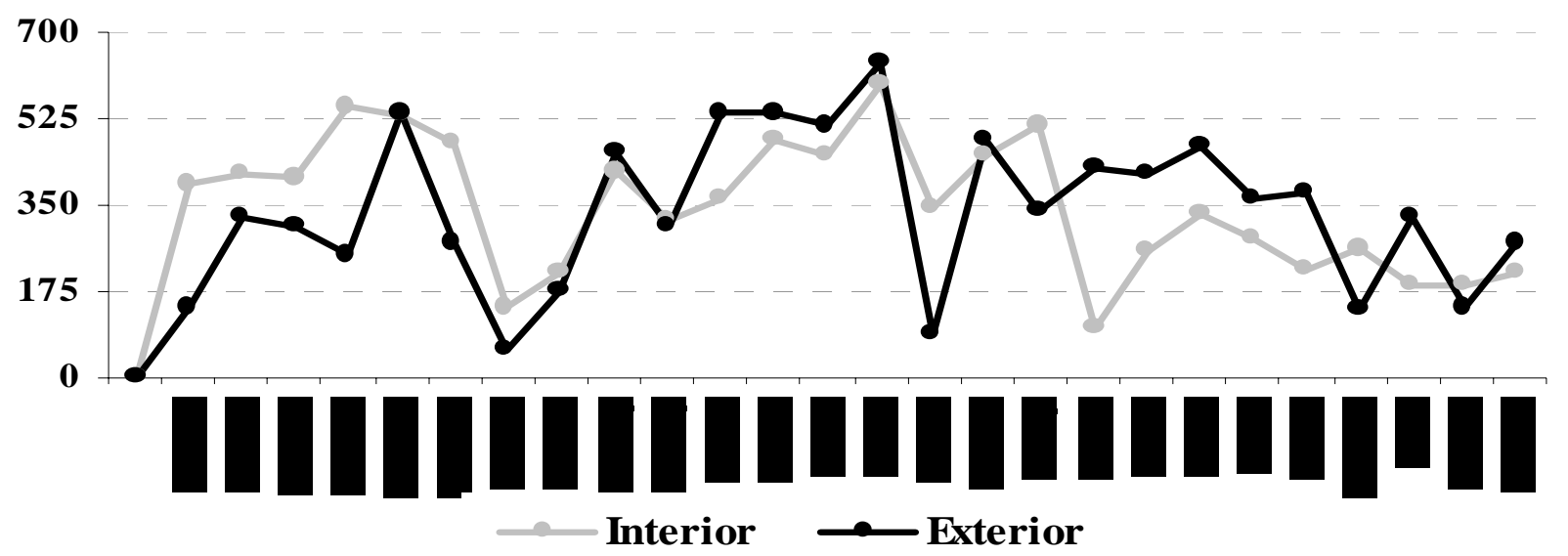

Figura 3.- Promedio de $\mathrm{CFU} / \mathrm{m}^{3}$ a lo largo del año

fúngicas cerca del mediodía, registrándose un promedio de $406 \mathrm{CFU} / \mathrm{m}^{3}$ a las $13 \mathrm{~h}$ frente a $379 \mathrm{CFU} / \mathrm{m}^{3}$ a las 9 h. y 334 $\mathrm{CFU} / \mathrm{m}^{3}$ a las $21 \mathrm{~h}$. Los valores obtenidos a las $13 \mathrm{~h}$, han sido superiores a los de las $9 \mathrm{~h}$, en nueve de los trece días muestreados y a los de las 21 h, en 10 de los 13 días muestreados. En ambos casos, las diferencias más importantes fueron superiores a $100 \mathrm{CFU} / \mathrm{m}^{3}$.

En la atmósfera exterior, por el contrario, las concentraciones de propágulos son superiores a las $9 \mathrm{~h}$ con promedio de $427 \mathrm{CFU} / \mathrm{m}^{3}$, frente a las $305 \mathrm{CFU} / \mathrm{m}^{3}$ a las $13 \mathrm{~h}$ y $385 \mathrm{CFU} / \mathrm{m}^{3}$ a las $21 \mathrm{~h}$. Al comparar los resultados interior/ exterior, lo más sobresaliente es la diferencia a las 13 h (406 $\mathrm{CFU} / \mathrm{m}^{3}$ interior versus $305 \mathrm{CFU} / \mathrm{m}^{3}$ exterior), coincidiendo con la afluencia masiva de personas al templo (A).

Para ver la correlación estadística entre los factores meteorológicos y la concentración fúngica en los diferentes puntos de muestreo, se aplicó el test de Spearman, resultando que en la Catedral, la temperatura explica el $21 \%$ de los casos y la humedad el $42 \%$ cuando la afluencia de gente era constante (B). En ambos casos la correlación fue positiva y el grado de confianza del test de un 99\%. En el exterior, el único parámetro que resultó con

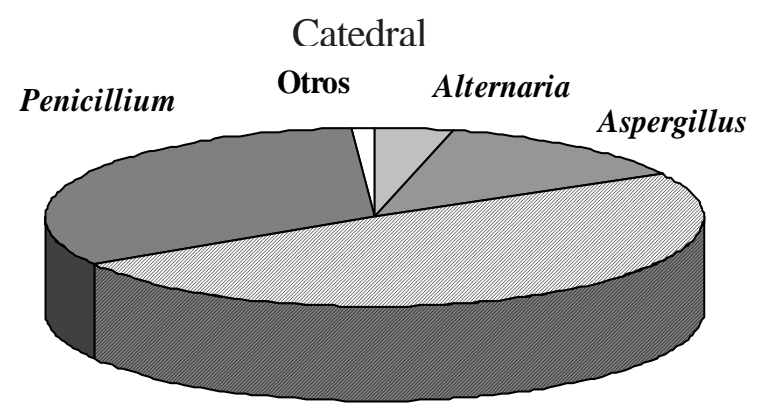

Cladosporium elevada significación estadística (95\% en este caso), ha sido la temperatura media, ya que explica el $31 \%$ de los casos y los correlaciona de forma positiva.

El análisis cualitativo ha permitido identificar 28 taxa de los cuales 20 corresponden a muestras tomadas en el interior de la Catedral y 22 de la atmósfera externa de un total de 1.974 aislamientos (Tabla 3).

La gran mayoría de las especies identificadas representan porcentajes muy bajos, con excepción de 4 géneros, Aspergillus, Cladosporium, Penicillium y Alternaria, dominando los tres primeros en el interior, mientras que en el exterior destaca Cladosporium (Figura 4).

La especie más abundante de Alternaria fue $\boldsymbol{A}$. alternata (Fr.) Keissl. mientras que en Cladosporium hay codominancia de $\boldsymbol{C}$. cladosporioides (Fr.) de Vries y $\boldsymbol{C}$. herbarum (Pers.) Link.

Si analizamos la representación intragenérica de Aspergillus, se observa que la especie más abundante fue $\boldsymbol{A}$. fumigatus Fres., representando un $65 \%$ en la atmósfera de la Catedral y un 63\% en el exterior y A. versicolor (Vuillemin) Tiraboschi, con un 20\% -22\% respectivamente.

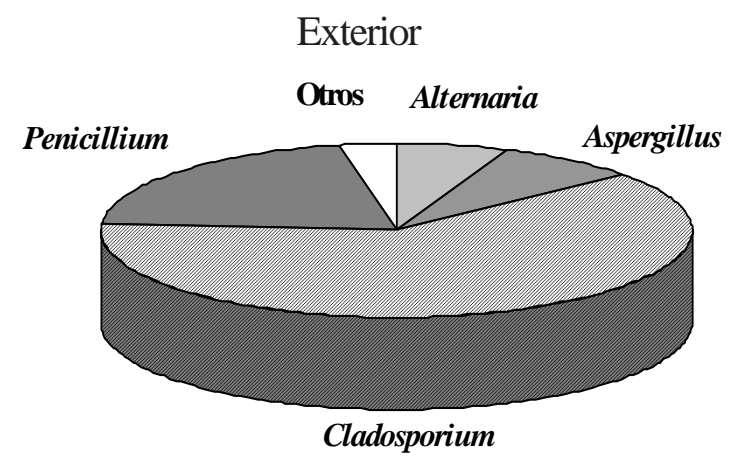

Figura 4 .- Promedio de CFU/ms de los principales taxa 


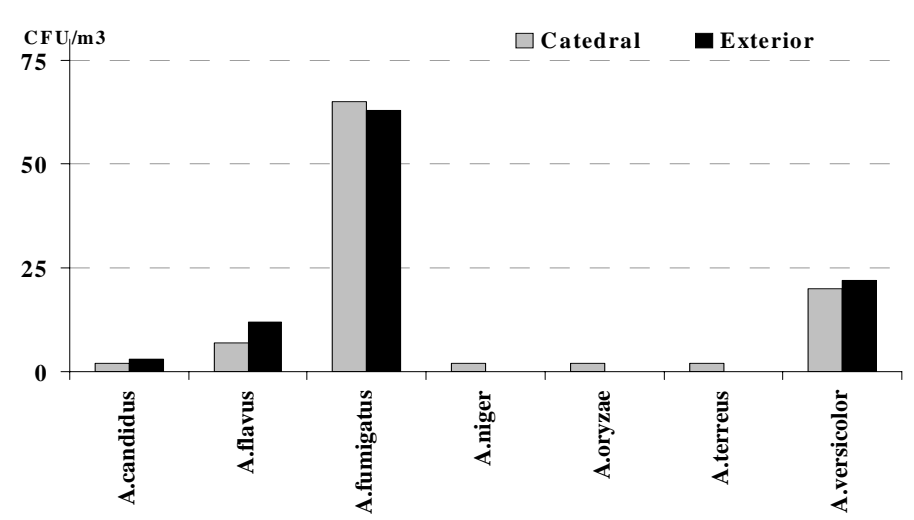

Figura 5 .- Representatividad intragenérica de Aspergillus

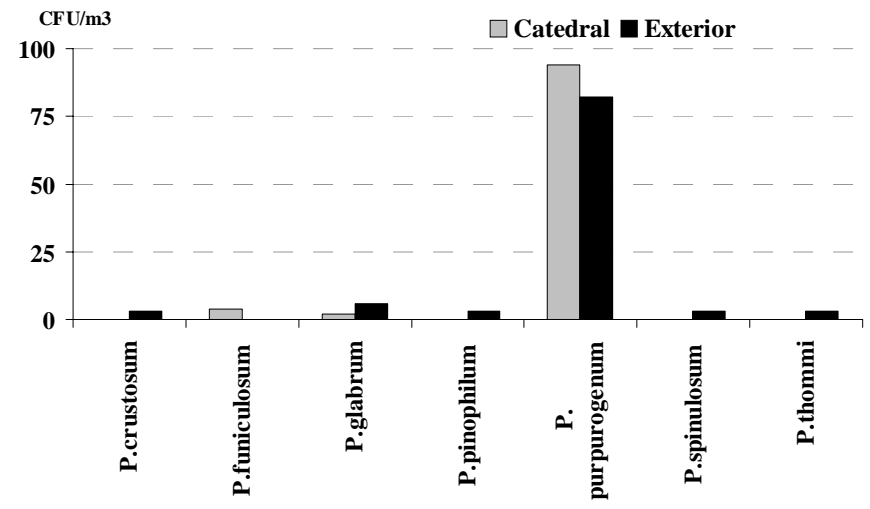

Figura 6 .- Representatividad intragenérica de Penicillium

Finalmente cabe resaltar la gran abundancia de Penicillium purpurogenum Stoll, especie que fue dominante dentro de su género con un $94 \%$ en la atmósfera interna y un 82\% en el exterior (Figuras 5 y 6).

\section{DISCUSION}

El estudio de la representatividad fúngica en la Catedral pone de manifiesto un valor promedio de unas $400 \mathrm{CFU} / \mathrm{m}^{3}$. Valores similares se han obtenido en el Archivo Estatal de Roma, donde los géneros predominantes han sido Cladosporium en invierno y Penicillium en verano, junto a otros hongos comunes en cualquier época del año como Alternaria, Aspergillus y Chaetomium (Maggi et al., 2000).

Los trabajos relacionados con la calidad del aire en el interior de edificios comenzaron a desarrollarse con fines sanitarios, tanto en viviendas como en escuelas y hospitales (Dales et al., 1991; Nunes et al., 2005), ya que muchas personas sufrían lo que se definió como el síndrome del edificio enfermo, causado principalmente por la alergia respiratoria a las esporas de A.fumigatus, Cladosporium, Alternaria y Stachybotrys. Aunque no hay datos estandarizados, se acepta que entre 10-100 CFU/ $\mathrm{m}^{3}$ son niveles normales en una atmósfera limpia (Kowalski, 2000) mientras que entre 150 a 1.000 CFU/ $\mathrm{m}^{3}$ son suficientes para causar problemas de salúd (Curtis et al., 2004). Por su parte, Stark et al. (2003), consideran que la importancia del nivel fúngico en un ambiente interno, varía según el tipo de hongo; así $100 \mathrm{CFU} / \mathrm{m}^{3}$ de Aspergillus es una cantidad elevada e incluso con menos Stachybotris puede resultar nocivo (Kuhn \& Ghannoum, 2003).

Las condiciones que favorecen la contaminación microbiológica del aire en ambientes internos, son la humedad elevada y la reducida ventilación, aunque siempre es necesario un sustrato que proporcione al hongo sus nutrientes (madera, celulosa, textiles varios, como cortinas, alfombras, etc). En el exterior, se ha señalado que la elevada temperatura y humedad, favorecen la presencia de esporas fúngicas en la atmósfera (Shelton et al., 2002).

Los hongos identificados en la Catedral de Santiago son comunes con los de otros recintos de las mismas características, como la Catedral de Moscú, donde los géneros más abundantes fueron Acremonium y Penicillium (Petushkova \& Kandyba, 1999) o los de frescos de diferentes monasterios italianos, donde se ha puesto de manifiesto la presencia de hongos como Alternaria, Aspergillus, Cladosporium, Fusarium, Penicillium y Rhizopus, siendo las especies más comunes C.cladosporioides, A. alternata, A. niger

y P. expansum (Guglielminetti et al., 1994; Pitzurra et al., 1999), en gran parte coincidentes con los resultados de un estudio realizado en diferentes edificios históricos alemanes donde además se identificaron géneros como: Acremonium, Aureobasidium, Beauveria, Chrysosporium, Engyodontium, Scopularopsis y Verticillium (Gorbushina et al., 2000). Nugari et al. (1993), en una recopilación bibliográfica de trabajos sobre biodeterioro, encontraron que los géneros más frecuentemente mencionados son Penicillium, Aspergillus, Cladosporium y Chaetomium, aunque el más severo es Cladosporium. El efecto de este hongo, junto con Rhizopus, Aspergillus y Fusarium, pueden alterar papeles, fotografías y planos de fibras textiles en 25 días (Mateus et al., 1999). Otros géneros como Trichoderma, Stachybotris, Stemphylium, Alternaria y Mucor, han sido citados como responsables de causar el biodeterioro de libros, objetos de arte, material audiovisual, pintura, papel tapiz, madera, murales y pieles (Gallo, 1993) y Acremonium, como uno de los hongos implicados en el proceso de biodeterioro de piezas de vidrio (Gorbushina \& Palinska, 1999). Por su parte, Aspergillus y Penicillium son dos de los géneros más prolíficos y ubicuos en todo el mundo, resultando con alta significa- 
ción en este muestreo. En su vertiente negativa para el hombre, pueden ocasionar patologías de diferente índole, en especial ciertos integrantes de género Aspergillus (De Hoog et al. 2000), algunas con pronóstico muy grave como es el caso de las infecciones invasivas llamadas en general aspergilosis, aunque los niveles aquí registrados parecen no alcanzar a un factor de riesgo. A. fumigatus, fue la especie más importante del género en la Catedral, se trata de un contaminante muy frecuente sobre materia orgánica húmeda. Es un termotolerante especial ya que su óptima es de $45^{\circ} \mathrm{C}$ y ha sido aislado de la atmósfera, caucho, materia orgánica en descomposición, material sintético, plástico, papel, alimentos, reservas de hidrocarburos, suelos, textil (algodón, lana, yute), etc. (Gams et al., 1985; De Hoog et al., 2000). Los conidios de $\boldsymbol{A}$. fumigatus, al entrar al tracto respiratorio pueden producir diversas manifestaciones clínicas; desde síntomas alérgicos en individuos atópicos o no atópicos y en inmunocomprometidos pueden causar infeciones superficiales, enfermedades saprofíticas, aspergilosis pulmonar y diseminarse a menudo a otros organos (Groll \& Walsh, 2001; Del Palacio et al. 2003).

En cuanto a Penicillium, es bien conocida la actividad de sus enzimas en la descomposición de materiales orgánicos como el papel, la madera y textiles (Sunna \& Antrainikian, 1997; Villalba et al., 2004). El aislamiento de $\boldsymbol{P}$. purpurogenum como la especie de más alta concentración en el interior y exterior de la Catedral, permite varios comentarios en relación a su ecología y aspectos fisiológicos: es un degradador de la madera ya que secreta al medio xilanasas que intervienen en la biodegradación del xilano, una de las principales hemicelulosas (Ghosh et al., 1999), por lo que su abundancia en la Catedral podría causar un efecto nocivo en los retablos y tallas.

Moody et al. (1999), en estudios de las respuestas de los hongos de la litera y del filoplano consideran a P.purpurogenum (como también A. fumigatus) sensibles a la radiación UV-B (290-315 nm), la cual puede reducir en ambas especies la germinación de sus mitosporas hasta en un 22-44\%. La mayor presencia de estos propágulos de dispersión en el interior, guardan una relación primaria con la presencia de diferentes substratos aprovechables, favoreciéndo su multiplicación por la menor exposición de radiación UV-B en el interior del templo.

De Hoog et al .(2006), compararon la distribución de la xerotolerancia fúngica con la capacidad oportunística invasiva de sus integrantes en los animales de sangre caliente y $\boldsymbol{P}$. purpurogenum, es considerado como muchos otros integrantes del género un xerotolerante $\left(\mathrm{a}_{\mathrm{w}}\right.$ mínima de 0,84), con un bajo nivel de bioseguridad (BSL 1) (De hoog et al. 2000), o sea con extremadamente raras posibilidades de producir cuadros de micosis, aún en pacientes con compromiso inmune. Sin embargo, Breton et al. (1998), reportan 2 casos de micosis pulmonares por este agente en pacientes con enfermedades hematológicas (aplasia medular) y hacen referencia a un caso anterior (Morin et al ,1986). Actualmente se describe también un caso de micosis diseminada en un perro (Zanatta et al, 2006). Este agente podría llegar a considerarse en el tiempo como un débil patógeno emergente en la poblacion humana de alto riesgo.

En los Eurotiales, la xero, halo y termotolerancia es un fenómeno común y los integrantes del subgénero Biverticillium contiene un buen número de especies que se aislan de suelos secos, salinos y calentados, donde pertenece P.marneffei, con el más alto nivel de bioseguridad (BSL 3) y P.purpurogenum, entre otros, todos ellos con buen crecimiento a $37^{\circ} \mathrm{C}$.

P.purpurogenum también ha sido aislado de papel, agua, sedimentos de estuarios, dunas, suelos cultivados, pintura, productos alimentarios para el hombre y los animales, así como de la atmósfera (Sawane \&Saoji, 2005). Se desarrolla también sobre telas de algodón y más lento sobre cuero y pergamino (Dalcero et al. 1998; Pitt, 2000; De Hoog et al., 2000).

Finalmente, la amplia distribución de P.purpurogenum en muchos ambientes y substratos diversos, nos lleva a considerar no solo las posibles reacciones alérgicas que los integrantes del género Penicillium pueden causar en las personas atópicas, sino la producción de algunos de sus productos metabólicos como la rubratoxina B producidas por P.purpurogenum. A pesar que la distribución de esta aún no está bien establecida, sus efectos tóxicos en animales y líneas de células humanas, han demostrado propiedades mutagénicas en embriones, teratogénicas en ratones, hipertrofia hepática y daños en el sistema inmmune aún en bajas dosis (Beasley, 1999; Sava, et al., 2004; Richetti et al., 2005; Nagashima et al., 2006).

Como conclusión final de este estudio, consideramos que en la Catedral es necesario mejorar las condiciones de limpieza, sobre todo en alfombras y textiles en general, así como reparar o sustituir algunas piezas de madera de los techos, en los que el deterioro producido por hongos es evidente. En cuanto a la alteración observada en la piedra de algunos muros y columnas, sería necesario realizar un estudio interdisciplinario para conocer los aspectos fisicoquímicos y tratar de eliminar la humedad (Pasanen et al., 2000).

\section{AGRADECIMIENTOS}

El presente trabajo fue subvencionado por la Consellería de Cultura, Comunicación Social y Turismo de la Xunta de Galicia (PGIDT01PAT38301PR). 


\section{REFERENCIAS}

Beasley, V. (1999). Veterinary Toxicology. Inter. Vet. Information Service. www.ivis.org

Breton. P.; Germaud, P.; Morin, O.; Audouin, A.F.; Milpied, N.; Harousseau, J.L. (1998) Rare pulmonary mycoses in patients with hematologic diseases- Rev. Pneumol. Clin. 54: 253-257

Calderón, R.F. (2004). Caracterización y tratamiento de microorganismos bioindicadores de contaminación y deterioro en restos óseos humanos custodiados por el Laboratorio de Antropología Física de la Universidad Nacional (Colombia). Exhumar 1:101-111

Curtis; L. ; Lieberman, A. ; Stark, M.; Rea, W. \& Vetter, M. (2004). Adverse Health Effects of Indoor Moulds. Journal of Australasian College of Nutritional \& Environmental Medicine, 23: $3-8$

Dalcero, A.; Magnoli, C.; Luna, M.; Ancasi, G.; Reynoso, M.M.; Chiacchiera, S.; Miazzo, R.; Palacio,G. (1998). Мусоflora and naturally occurring mycotoxin in poultry feeds in Argentina. Mycopathologia 141: 37-43

Dales, R.E.; Zwanenburg, H.; Burnett, R. \& Franklin, C.A. (1991). Respiratory Health effects of home dampness and molds among canadian children. American J. of Epidemiol.134: 196203

Del Palacios, A.; Cuétaro, M.S. \& Pontón, J. (2003). La aspergilosis invasiva. Rev. Iberoam. Micol. 20: 77-78

Gallo, F. (1993). Aerobiological research and problems in libraries. Aerobiología. 9(2-3). 11-29

Gams, W., Christensen, M., Onions, A.H., Pitt, J.I. \& Samson, R.A. (1985).- I. Infrageneric taxa of Aspergillus. In: Advances in Penicillium and Aspergillus systematics (Eds. R.A. Samson \& J.I. Pitt), New York Plenum Press.pp: 55-62.

Ghosh, D.; Erman, M.; Sawicki, M.; Lala, P.; Weeks, D.R.; Li, N.; Pangborn, W., Thiel, D.J.; Jörnvall, H.; Gutiérrez, R. \& Eyzaguirre, J. (1999). Determination of a protein structure by iodination: The structure of iodinated acetylxylan esterase. Acta Crystallographica, Serie D 55: 779-784

Gorbushina, A.A. \& Palinska, K.A. (1999).- Biodeteriorative processes on glass: experimental proof of the role of fungi and cyanobacteria. Aerobiologia 15: 183-191.

Gorbushina, A.A.; Heyrman, J.; Dornieden, T.; Gonzalez del Valle, M.; Krumbein, W.E.; Laiz, L.; Petersen, K.; SaizJimenez, C. \& Swings, J. (2004). Bacterial and fungal diversity and biodeterioration problems in mural painting environments of St. Martinschurch (Greene-Kreiensen,, Germany). Int. Biodeter. Biodeg. 53: 13-24

Gorbushina, A.A.; Heyrman, J.; Mergaert, J.; Krumbein, WE \& Swings, J. (2000). Comparison of the microflora present on three severely damaged mural paintings subjected to different climatic conditions. $5^{\text {th }}$ Symposium on the Conservation of monuments in the Mediterranean Basin, april 5-8, Seville, Spain

Groll, A. H. \& Walsh, T.J. (2000). Aspergillosis. in Atlas of infectious diseases. Fungal infections (Mandell, G. L. \& Diamond, R.D. eds.) Current Medicine, Inc. Philadelphia. pp.136-160
Guglielminetti, M.; De Giuli, C.; Radaelli, A. ; Bistini, F. ; Carruba, G. ; Spera, G. \& Caretta, G. (1994). Mycological and ultrastuctural studies to evaluate biodeterioration of mural paintings. Detection of fungi and mites in frescos of the Monastery of St. Damian in Assisi. Inter Biodet \& Biodegr. 269-283.

Hoog de, G.S.; Guarro, J; Gené, J. \& Figueras, M.J. (2000) Atlas of Clinical Fungi. $2^{\text {a }}$ Eds.CBS, Universitat Roviri i Virgili. Reus. Eapaña.

Hoog, G.S.de.; Zalar, P.; VAn Den Ende, B.G.\& GundeCimerman, N. (2006). Relation to Halotolerance to humanpathogenicity in the fungal Tree of life: An overview of ecology and evolution Under Stress. http:/www.blackyeast.org/pdf/HaloSlovenia.pdf

Koestler, R.J. \& Santoro, E.D. (1987). Preliminary scanning electron microscopy study of microbiologically induced deterioration of high alkali low-lime glass. Biodeterioration Research 295-307

Kowalski, W.J. (2000). Indoor mold growth: health hazards and remediation. HPAC Engineering, 72: 80-83

Kuhn, D. M. \& Ghannoum, M. A., (2003). Indoor mold, toxigenic fungi, and Stachybotrys chartarum: infectious disease perspective. Clinic Microbiology Reviews, 16: 144-172

Maggi, O.; Persiani, A.M.; Gallo, F.; Valenti, P.; Pasquariello, G.; Sclocchi, M.C. \& Scorrano, M. (2000). Airborne fungal spores in dust present in archives: proposal for a detection method, new for archival materials. Aerobiologia, 16: 429-434.

Mateus, J; Peña, D.; Peña, G.; Rojas, A.; Rojas, J.; Zambrano, S.; Martínez, M.M.; Flórez, C. \& Santander, M. (1999). Seguimiento y control de biodeterioro microbiológico en documentos de interés histórico en el archivo general de la nación. Revista de la Facultad de Ciencias Pontificia Universidad Javeriana 9: $37-46$

Medina, L.; Tuozzo, A.; Herrera, J.; Perozo, Y. \& González, L. (1999). Estudio de hongos en bibliotecas de la Universidad de Carabobo-Valencia, vol. $3, \mathrm{n}^{\circ} 1$

Moody, S.A.; Newsham ,K.K.; Aires, P.G. \& Paul, N.D. (1999). Variation in the responses of litter and phylloplane fungi tu UV-B radiation (290-315 nm). Mycol. Res. 103-1469-1477

Morin, O.; Germaud,P.; Miegeville, M. \& Milipied, N.(1986). Mycose pulmonaire à Penicillium purpurogenum, à propos d'une observation chez a malade inmunodéprimé. Bull. Soc. Fr. Mycol.Méd. 15:441448

Nagashima, H.; Maeda-Nakamura, K.; Iwashita, K. \& Goto.T. (2006). Induced secretion of insulin-like growth factor binding protein-1 (IGFBP-1) in human hepatoma cell HepG2 by rubratoxin B. Arch.Toxicol. 16: (Epub ahead of print)

Nugari, M; Realini, M; Roccardi, A. (1993). Contamination of mural paintings by indoor airborne fungal spores. Aerobiología. 9: $131-39$

Nunes, Z.G.; Martins, A.S.; Altoe, A.L.; Nishikawa, M.M.; Leite, M.O.; Aguiar, P.F. \& Fracalanzza, S.E. (2005). Indoor air microbiological evaluation of offices, hospitals, industries, and shopping centers. Mem. Inst. Oswaldo Cruz, 100:321-330 
Pasanen, A.L.; Rautiala, S.; Kasanen, J.P.; Raunio, P.; Rantamaki, J. \& Kalliokoski, P. (2000). The relationship between measured moisture conditions and fungal concentrations in water-damaged building materials. Indoor Air, 10: 111-120

Petushkova, J. \& Kandyba, P. (1999). Aeromicrobiological studies in the Moscow cathedrals. Aerobiologia 15: 193-201.

Pitt, J.I. (2000). A laboratory guide to common Penicillium species. Food Science Australia, third Edition.

Pitzurra, L., Belleza, T.; Giammarioli, M.; Giraldo, M.; Sbaraglia, G. Spera, G. \& Bistoni, F. (1999). Microbial environmental monitoring of the refectory in the monastery of St. Anna in Foligno, Italy. Aerobiologia 15: 203-209

Richetti, A.; Cavallaro, A.; Ainis, T. \& Fimiani, V. (2005). Effect of mycotoxins on some activities of isolated human neutrophils. Immunopharmacology and Immunotoxicology 27:433-446

Saiz-Jimenez, C., Brimblecombe, P., Camuffo, D., Lefevre, R.A. \& van Grieken, R. (1999). Damages caused to European monuments by air pollution: assessment and preventive measures. Air Pollution and Cultural Heritage. Balkema Pub. 91-109

Sava, V.; Mosquera, D.; Song,S.; Stedeford, T.; Calero, K.; Cardozo-Pelaez, F.; Harbison, R.; Sanchez- Ramos, J. (2004). Rubratoxin B elicit antioxidative and DNA repair response in mouse brain. Gene Expression 11: 211-219

Sawane, A.M. \& Saoji, A.A. (2005). Airborne Penicillium in the grain shops of Nagpur (India).Grana 44:123-128

Shelton, B.G.; Kirkland, K.H.; Flanders, W.D. \& Morris, G.K. (2002). Profiles of airborne fungi in buildings and outdoor environments in the United States. Appl Environ Microbiol. 68: $1743-1753$
Singh, A.; Ganguli, M \& Singh, A.B. (1995). Fungal spores are an important component of library air. Aerobiologia 11: 231-237

Stark, P.C.; Burge, H.A.; Ryan, L.M.; Milton, D.K. \& Gold, D.R. (2003). Fungal levels in the home and lower respiratory tract illnesses in the first year of life. Am J Respir Crit Care Med., 168: 232-237

Stchigel, A.M.; Jato, V. \& Aira, M.J. (2002) Fungal taxa in the Cathedral and Museum of Santiago de Compostela (Spain). $7^{\text {th }}$ International Congress on Aerobiology, Aerobiology: Coming of age in a New Millenium. Chateau Montebello. Canadá

Sunna, A. \& Antrainikian, G. (1997). Xilanolitic enzymes from fungi and bacteria. Critical Reviews in Biotechnology 17: 39-67

Tapia, S.T. (2006).- ¿¿Bacterias cinéfilas? Encuentros en la Biología, $n^{\circ}$ 111: 3-4

Vaillant. C. M. \& Valentin, N. (1996). Principios básicos de la conservación documental y causas de su deterioro. Monografías del Ministerio de Educación y Cultura, España

Villalba, L.S.; Mikán, J.F. \& Sánchez, J. (2004). ctividades hidrolíticas y caracterización isoenzimática de poblaciones microbianas aisladas del patrimonio documental del Archivo Generad de Colombia. Nova Publicación Científica 2 (2): 50-57anatta, R.;

Zanatta, R.; Miniscalco, B.; Guarro, J.; Gené, J.; Capucchio, M.T.; Gallo, M.G.; Mikulicich, B.; Peano, A. (2006). A case of disseminated mycosis in a German Shepered dog due to Penicillium purpurogenum. Medical Mycology 44:93-97 